\title{
Des souris transgéniques porteuses d'un gène muté de collagène, un modèle d'Osteogenesis imperfecta
}

Le collagène de type I est une protéine majeure de la matrice extracellulaire. Chaque molécule est formée de deux chaînes $\alpha$ l[1] et d'une chaîne $\alpha 2$ [1], qui, à la suite de coupures protéolytiques aux deux extrémités du précurseur, s'enroulent en une triple hélice. La région en hélice est faite de la répétition de triplets gly-XXX (X étant souvent une proline). La molécule subit des modifications post-traductionnelles, glycosylation et surtout hydroxylation de prolines et de lysines, qu'interrompt la constitution de la triple hélice. Des anomalies du collagène de type I sont observées dans plusieurs formes d'une maladie héréditaire, l'Osteogenesis imperfacta (OI). La forme la plus sévère, dite de type II, est une maladie dominante autosomique, causant la mort in utero ou peu de temps après la naissance. Le collagène de type I est diminué et «surmodifié»: l'hydroxylation des lysines et la glycosylation sont excessives, probablement parce que la triple hélice tarde à se former, ce qui expose la molécule a une action prolongée des enzymes d'hydroxylation. La lésion biochimique du gène est, soit une délétion, soit une mutation ponctuelle, conduisant au remplacement d'un glycocolle par une cystéine ou par une arginine.

Une équipe unissant des chercheurs de Boston et de Melbourne[1] a voulu reproduire expérimentalement cette dernière lésion chez la souris. Par mutagenèse dirigée, ils ont produit un clone génomique codant pour une protéine portant en position 859 une cystéine ou une arginine à la place d'un glycocolle. Ils ont d'abord transfecté cette construction dans des cellules en culture de deux types: la classique lignée NHI $3 T 3$, qui contient le gène normal de collagène $\alpha$ l [1], et des fibroblastes homozygotes Movl3, qui ne produi- étudier l'expression du gène muté soit seul, soit en présence du gène normal. Dans tous les cas, il est possible de détecter les chaînes mutées, produites en quantités variables, et qui ont une migration ralentie à l'électrophorèse, témoin de l'augmentation de l'hydroxylation et de la glycosylation.

La génération in vivo de souris transgéniques porteuses du gène muté a donné des résultats impressionnants. De l'ADN provenant d'un cosmide contenant le gène muté gly $\rightarrow$ cys avec son promoteur a été injecté dans des œufs fécondés puis réimplantés. Sur 32 souris viables à la naissance, aucune ne portait le transgène. En revanche, sept des neuf souris mortes en période périnatale (juste avant ou après la naissance) le contenaient. Un transgène normal humain ou murin ne créait aucun dommage. Les animaux atteints étaient flaccides, leur ossification retardée. Leur peau contenait du collagène muté en quantité variable, avec une baisse considérable du collagène total de type I malgré la présence des gènes normaux.

L'expression du gène muté chez les souris transgéniques provoque donc l'apparition d'un phénotype létal dominant ressemblant cliniquement et biochimiquement à celui de l'OI humaine. Il s'agit d'une conséquence directe de la mutation, qui rompt la séquence des triplets gly-X-X. De plus, la présence du produit anormal retentit sur l'organisation et la stabilité du collagène produit par le ou les gènes normaux. Il devient ainsi possible d'étudier sur un modèle animal les effets et les mécanismes de maladies dominantes.

J.-C. D.

1. Stracey A, Bateman J, Choi T, Mascara T, Cole W, Jaenisch R. Perinatal Osteogenesis imperfecta in transgenic mice bearing an engineered mutant pro- $\alpha 1$ (1) collagen gene. Nalure $1988 ; 332$ : 131-6.

\section{BRĖVES}

Absence de récepteurs du TGF- $\beta$ dans le rétinoblastome. Le TGF- $\beta$ (transforming growth factor) est, selon les cellules, un facteur de croissance (pour les fibroblastes principalement) ou, le plus souvent, un inhibiteur de la prolifération cellulaire $\left(\mathrm{m} / \mathrm{s} n^{\circ} 8\right.$, vol. 2 , p. 467). Cela est particulièrement net au niveau des cellules rétiniennes normales [1]. Au contraire, les cellules de rétinoblastome semblent être insensibles à l'effet antiprolifératif du TGF- $\beta$. De plus, trois glycoprotéines de 65,95 et $300 \mathrm{kDa}$, présentes dans les cellules normales et ayant une forte affinité pour le TGF- $\beta$ sont absentes dans les cellules de rétinoblastome. On peut supposer que l'absence de récepteur pour le TGF- $\beta$ fait perdre aux cellules de rétinoblastome leur sensibilité à un contrôle négatif de la prolifération. Les rapports entre l'absence du récepteur du TGF- $\beta$ et l'anomalie du gène $\mathrm{RB}$ de sensibilité au rétinoblastome restent inconnus (m/s $n^{\circ} 6$, vol. 3, p. 363).

[1. Kimchi A, et al. Science 1988 240: 196-9.]

Structure du récepteur de la prolactine et similitudes avec celui de l'hormone de croissance. La structure du récepteur de la prolactine de rat a été déduite de la séquence nucléotidique de son ADN complémentaire par une équipe québeco-française [1]. Il s'agit d'une molécule de taille relativement petite $(40000$ daltons), transmembranaire, semblant appartenir à la même famille que le récepteur de l'hormone de croissance, récemment cloné ( $\mathrm{m} / \mathrm{s}$, vol. $4, p .324)$ avec de courts segments protéiques très homologues dans les deux molécules. Contrairement au récepteur de l'hormone de crọissance, celui de la prolactine a une très courte région intracytoplasmique; dans les deux cas la structure ne révèle aucun domaine susceptible d'être doté d'une activité de protéine kinase.

[1. Boutin JM, et al. Cell 1988; 53 : 69-77.] 
Une même protéine provoque une différenciation érythroïde et contrôle la sécrétion de FSH. Des auteurs japonais [1] ont isolé un polypeptide doté d'une puịsante activité inductrice de différenciation érythroïde sur les cellules de Friend de souris et sur la lignée humaine K 562. La séquence du messager et de la protéine dérivée se sont révélées identiques à celles d'une chaîne polypeptidique déjà connue. Il s'agit d'une glycoprotéine ovarienne, exerçant une action régulatrice sur la sécrétion pituitaire de la FSH. Cette régulation, curieusement, se fait dans les deux sens: lorsque cette sous-unité, baptisée $\beta$ A, s'unit à ellemême pour former un homodimère, elle active la sécrétion; elle l'inhibe au contraire lorsqu'elle se lie à une sous-unité $\beta B$ pour former un hétérodimère qui pour cette raison a été appelé inhibine. Ce travail fournit un nouvel exemple de facteurs, découverts indépendamment par des groupes différents, possédant des activités très diverses, et qui, à l'analyse, se révèlent identiques.

[Murata M, et al. Proc Natl Acad Sci USA 1988 ; 85 : 2434-8.]

Transfert, par l'intermédiaire d'un vecteur rétroviral, du gène de $\beta$ globine humaine dans des cellules progénitrices de la lignée rouge. Un nombre important d'hémoglobinopathies graves ( $\beta$-thalassémie et drépanocytose) est dû à des désordres structuraux au niveau des gènes de globine. Des travaux expérimentaux ont par ailleurs montré que le gène de $\beta$-globine humaine introduit dans des cellules étrangères ou des souris transgéniques est soumis à une régulation normale. Son introduction dans des cellules érythropoïétiques a donc été envisagée comme une approche thérapeutique. Le vecteur envisagé est un rétrovirus dans lequel est inséré un gène de $\beta$-globine et qui peut lui-même être introduit et incorporé dans une cellule érythroïde. Afin de tester cette incorporation, le gène $\beta$-globine a été légè- rement modifié dans la région 5 ' non codante par une insertion créatrice d'un site de restriction qui ne modifie pas son expression, mais permet de le différencier du gène endogène. Le vecteur et le gène ont d'abord été introduits dans des cellules érythroleucémiques de souris, ce qui a permis de démontrer la nécessaire présence des introns pour obtenir une expression quantitativement et qualitativement normale. Dans ces cas le niveau d'expression approchait celui observé pour le gène endogène (une régulation normale qui, dans certains cas, atteint le même niveau que celui de la globine endogène de souris). Il a ensuite été mis en présence de culture de cellules progénitrices humaines, BFUe. La fréquence de l'infection a été faible dans les premières expériences, mais le taux d'expression par rapport au gène endogène est d'environ $5 \%$ pour un seul gène incorporé. $\mathrm{Si}$ l'on considère qu'un taux de $20 \%$ de chaîne $\beta$ normale est, en règle, suffisant pour assurer un caractère intermédiaire à une $\beta$-thalassémie, il est possible d'espérer une amélioration des résultats, surtout si l'on pouvait transférer, avec le gène, des séquences enhancer ( $m / s n^{\circ} 4$, vol. 4, p. 252). Ceci ouvrirait les perspectives d'une thérapeutique génique

[Bender MA, et al. Mol Cell Biol $1988 ; 8: 1725-35$.]

Les ribozymes et l'origine de la vie. La découverte que des molécules d'ARN pouvaient être douées d'activités enzymatiques (auto-épissage des introns, activité ribonucléasique, voir m/s suppl. au $n^{\circ} 7$, vol. 3, p. 30) a immédiatement suggéré qu'une des premières molécules de la vie ait pu être un ARN autoréplicatif et autocatalytique. T. Cech vient de réaliser une expérience confortant cette hypothèse [1]: en présence de l'intron de Tetrahymena thermophila (intron capable de s'auto-exciser et de s'autocycliser) et de dinucléotides comportant un $\mathrm{G}$ en 5', une amorce d'acide pentaribocytidylique peut être allongée en l'absence de toute protéine. L'ARN intronique catalytique joue donc le rôle d'une enzyme (d'où le terme proposé de ribozyme) pouvant figurer ce qu'était la «replicase » originelle.

[1. Been MD, Cech TR, Science $1988 ; 239$ : 1412-6.]

Le fibroblast growth factor (FGF) prévient in vivo la mort de neurones cholinergiques après section de leur axone, comme cela avait été démontré précédemment pour le nerve growth factor (NGF). L'équipe de Carl Cotman de l'Université d'Irvine en Californie, qui travaille depuis une dizaine d'années sur les facteurs trophiques agissant dans le cerveau des mammifères, vient d'établir que l'injection dans les ventricules cérébraux de très faibles quantités de FGF permet de préserver plus de $80 \%$ des cellules du cerveau antérieur qui produisent de l'acétylcholine lorsqu'on sectionne expérimentalement leur axone (au lieu de $50 \%$ environ de survie post-lésionnelle sans traitement). Cette proportion de survie est comparable à celle observée lorsqu'on injecte du NGF. On avait démontré jusqu'à présent l'action " throphique » du FGF sur les neurones (comme de l'epidermal growth factor, EGF ; voir $\mathrm{m} / \mathrm{s} n^{\circ} 1$, vol. 4 , p. 61) dans des expériences in vitro, mais il restait à démontrer son efficacité chez l'animal vivant. Voilà qui est fait, et cela ouvre peut-être des perspectives non seulement scientifiques mais aussi thérapeutiques. FGF existe en effet dans les zones cérébrales où se projettent normalement les axones des neurones cholinergiques; on peut donc penser que cette substance agit, hors de toute lésion cérébrale, dans le maintien des connexions. Mais de plus, on incrimine la faillite de l'innervation cholinergique corticale dans certains aspects de la maladie d'Alzheimer. La possibilité d'une préservation de neurones cholinergiques grâce à une action thérapeutique du FGF est donc très sérieusement envisagée.

[Anderson KJ, et al. Nature 1988 ; 332 : 360-1]

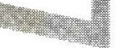


La représentation du corps par le cerveau de l'adulte se modifie en fonction des stimulations tactiles reçues. Dans le cortex cérébral, l'aire qui reçoit les messages induits par le toucher d'une partie de la surface cutanée (l'aire somesthésique primaire) est organisée suivant une topographie très stricte qui à chaque région du corps fait correspondre un petit groupe de neurones. Mike Merzenich de l'Université de Californie à San Francisco s'est posé la question du devenir de cette topographie lorsque la surface cutanée est modifiée cher. des singes adultes. Il a ainsi montré [1] que lorsqu'on coupe un doigt à un singe, l'aire somesthésique primaire «répond » à l'absence de cette partie de la surface cutanée par une récupération, au profil des doigts adjacents, des neurones libérés de leur relation précédente. En l'absence du majeur, les neurones activés précédemment à partir de ce doigt répondent au toucher, soit de l'index, soit de l'auriculaire. Cette plasticité de la topographie corticale était déjà tout-à-fait surprenante compte tenu de l'apparente rigidité des connexions dans le système nerveux central du mammifère adulte.La même équipe démontre aujourd'hui [2] qu'il existe un phénomène équivalent de plasticité lorsqu'on colle pour un temps assez long deux doigts ensemble. Dans ce cas, les neurones de l'aire somesthésique primaire précédemment liés spécifiquement à l'un ou à l'autre des deux doigts collés se mettent à répondre à des stimulations tactiles appliquées indifféremment à l'un ou l'autre des deux doigts. Cette réponse persiste pendant plusieurs jours si l'on sépare à nouveau les deux doigts, ce qui valide l'hypothèse d'une réelle plasticité des connexions nerveuses dans le cerveau adulte. D'un point de vue théorique, cette expérience ouvre en fait une nouvelle perspective, celle du lien entre l'activité et la représentation corticale. On peut en effet considérer que c'est la simultanéité des messages envoyés à partir des deux doigts collés qui provoque au cours du temps cale. La stimulation tactile, «l'utilisation du système somesthésique», modifierait donc les connexions nerveuses dans l'aire de réception corticale. Mais dans ce cas, on peut s'interroger sur la «quantité » de stimulation nécessaire à ce changement? Chaque stimulation tactile prise isolément n'entraînerait-elle pas normalement, en fait, en même temps que l'activation d'un groupe de neurones, une micro-altération de la topographie, altération dont l'effet ne se révèlerait à nos instruments d'analyse qu'avec la répétition de la même stimulation cutanée? Une telle hypothèse, qui a été directement présentée par M. Merzenich dans un récent congrès, nous conduit à l'image d'un cerveau en perpétuel mouvement, fort loin du cadre rigide imposé jusque-là.

[1. Merzenich MM, et al. J Comp Neurol 1984; 224: 591-605]

[2. Clark SA, et al. Nature $1988 ; 332$ : 444-5]

- Syndrome de l'homme raide et auto-immunité. Le syndrome de l'«homme raide» (stiff man syndrome) est une maladie rare, qui fit l'objet de nombreuses publications il y a 30 ans. Apparition à l'âge adulte, raideur du tronc et des parties proximales des membres, spasmes douloureux, forment l'essentiel des symptômes. Deux éléments ont permis d'orienter les recherches: l'action thérapeutique du diazépam qui a fait soupçonner l'atteinte de voies inhibitrices du tonus musculaire, en provenance du tronc cérébral et favorisées par le GABA ; et la découverte, chez certains malades, de quantités excessives d'IgG dans le liquide céphalorachidien (LCR). Solimena et al., à Milan[1] ont démontré dans le sérum et le LCR d'une malade la présence d'anticorps contre une enzyme, la décarboxylase de l'acide glutamique (GAD), enzyme responsable de la biosynthèse du GABA. Des coupes de cerveau incubées avec le sérum ou le LCR du malade montraient une réaction intense identi- que à celle obtenue avec un ant icorps anti-GAD ; de plus, l'électrophorèse de protéines cérébrales, suivie de réaction avec le sérum, montrait l'identité de la protéine réactive et de la GAD. La seule autre réponse à l'anticorps était donnée par les cellules $\beta$ des îlots de Langerhans du pancréas, connus pour contenir de la GAD, chez cette malade porteuse d'un diabète d'apparition récente. De ces travaux on peut conclure que cette malade - ainsi qu'un autre en cours d'étude - possède des autoanticorps, parmi lesquels un anticorps contre la GAD pourrait être responsable des symptômes neuromusculaires. Ce serait [2] le premier exemple d'une maladie affectant le système nerveux central due à un auto-anticorps dirigé contre une enzyme spécifique.

[1. Solimena M, et al. $N$ Engl J Med $1988 ; 318$ : 1012-20.]

[2. Layzer RB. N Engl J Med 1988 ; 318: 1060-1.]

C'est l'imperméabilité de la membrane nucléaire qui semble limiter la réplication de l'ADN. L'ADN se réplique précisément une fois et une seule par cycle cellulaire. Dans un système de réplication in vitro dérivé d'oeufs de Xenopus, des noyaux venant de subir une réplication, et transférés dans un extrait neuf, ne se répliquent pas en présence de cyclohéximide, un inhibiteur de la synthèse protéique. Cependant, l'addition d'une substance provoquant une dégradation de la membrane nucléaire (le MPF, maturing promoting factor) permet, en l'absence de synthèse protéique, la reprise de la réplication. Si la synthèse protéique n'est pas bloquée, la réplication reprendra lorsque la membrane nucléaire se dissociera. Ces résultats suggèrent que l'imperméabilité de la membrane nucléaire, limitant l'accès au noyau de substances indispensables, joue un rôle central dans le contrôle de la réplication de l'ADN.

[Blow J], Laskey RA. Nature 1988; 332 : 546-8.] 